\title{
Oral Pathergy in Sweet's Syndrome Following Food Bolus Injury
}

\author{
David Klimpl, Thomas Manser, Mark Flemmer \\ Department of Internal Medicine, Eastern Virginia Medical School, Norfolk, USA \\ Email: david.klimpl@gmail.com
}

Received 28 February 2016; accepted 18 April 2016; published 21 April 2016

Copyright @ 2016 by authors and Scientific Research Publishing Inc.

This work is licensed under the Creative Commons Attribution International License (CC BY).

http://creativecommons.org/licenses/by/4.0/

c) (i) Open Access

\begin{abstract}
Pathergy is a unique and dramatic clinical finding of exuberant inflammation in response to local trauma. This incompletely understood phenomenon presents with sterile pustules and ulcers after minor cuts or scrapes, and can be tested by a pricking the skin with a sterile needle. Historically, pathergy was thought to be pathognomonic for Behcet's syndrome, though it has also been described in several other inflammatory conditions such as pyoderma gangrenosum and acute febrile neutrophilic dermatosis (Sweet's syndrome). Recognizing Sweet's syndrome specifically can be challenging due to its atypical clinical course, and understanding the relationship between Sweet's syndrome and pathergy can offer an important diagnostic clue. We present a case of Sweet's syndrome presenting with upper airway obstruction and pathergy. To our knowledge, this is the first documented case of Sweet's syndrome presenting with oral pathergy.
\end{abstract}

\section{Keywords}

Pathergy, Sweet's Syndrome, Myelodysplastic Syndrome, Neutrophilic Dermatosis

\section{Introduction}

Sweet's syndrome, or acute febrile neutrophilic dermatosis, is a rare, poorly understood systemic inflammatory disorder. Originally described in 1964 by Dr. Robert Sweet, this syndrome presents with abrupt onset of erythematous or hemorrhagic rash with skin biopsy showing neutrophilic invasion of the dermis without leukocytoclastic vasculitis [1]. The presences of fever, leukocytosis, elevated inflammatory markers, and rapid resolution with prednisone are also diagnostic characteristics [Table 1]. Most cases are idiopathic, but a significant percent are either drug induced (most commonly granulocyte colony stimulating factor) or paraneoplastic (solid organ or hematologic) [2]. The pathergy phenomenon has also been associated with Sweet's syndrome, most often associated with the paraneoplastic subtype. We present the case of a 68 years old female who presented to the hospital with upper airway obstruction from pathergy and was found to have Sweet's syndrome. 
Table 1. Diagnostic criteria for Sweet's syndrome. The presence of both major and two minor criteria are required.

\begin{tabular}{|c|c|}
\hline Major criteria & Minor criteria \\
\hline $\begin{array}{l}\text { Abrupt onset of characteristic rash } \\
\text { (papules and nodules) }\end{array}$ & Fever $>38$ degrees Celsius \\
\hline \multirow[t]{3}{*}{$\begin{array}{l}\text { Biopsy proven dermal infiltration of } \\
\text { neutrophilswithout vasculitis }\end{array}$} & $\begin{array}{l}\text { Association with malignancy or } \\
\text { inflammatory syndrome }\end{array}$ \\
\hline & Excellent response to glucocorticoids \\
\hline & $\begin{array}{ll} & \text { Elevated inflammatory markers } \\
\text { on presentation (3 of } 4 \text { ) } \\
\text { - } & \text { ESR }>20 \mathrm{~mm} / \mathrm{h} \\
\text { - } & \text { Positive C-reactive protein } \\
\text { - } & \text { Leukocyte count }>10,000 / \mathrm{ul} \\
\text { - } & \text { Over } 70 \% \text { neutrophils }\end{array}$ \\
\hline
\end{tabular}

Source: Malone et al., Arch Dermatol. 2002; 138(3): 345-349. doi:10.1001/archderm.138.3.345.

\section{Case Report}

A 68 years old Asian female with a past medical history of transfusion dependent myelodysplastic syndrome (MDS) presented with airway obstruction from acute oropharyngeal swelling. The patient was emergently nasally intubated for airway protection and was therefore unable to give a history, but per her family she experienced 5 days of fever, oral pain, and facial edema prior to admission. She was febrile to 39.1 degrees Celsius, tachycardic to 147 beats per minute, and mildly hypertensive to $144 / 87 \mathrm{mmHg}$. Physical exam showed a diffusely edematous oropharynx and tongue with scant hemorrhagic bullae. The remainder of her physical exam was normal. Her CBC, BMP and inflammatory markers are shown on Table 2.

CT soft tissue neck revealed a $2.7 \times 1.4 \times 3.8 \mathrm{~cm}$ mass along the R palate and R neck. Vancomycin, clindamycin, and meropenem were started empirically, along with IV dexamethasone $4 \mathrm{mg}$ q6h. Biopsy of the oral process showed inflammation with necrosis and hemorrhage, but no neoplasia. Blood and tissue cultures were negative. An autoimmune workup was performed and was negative for lupus, mixed connective tissue disease, rheumatoid arthritis, Sjogrens, vasculitis, or hereditary angioedema [Table 3].

The patient's swelling \& fever improved and she was extubated hospital day four, and steroids were tapered off. Two days later, she developed recurrent fevers, oral pain and swelling. Repeat blood cultures were negative, and buccal biopsy staining and culture showed no bacteria, AFB, fungus, or neoplasm. Microscopy of the buccal mass tissue was again consistent only with chronic and acute hemorrhage with scattered mast cells and neutrophils. The return of fever after discontinuation of glucocorticoids, superimposed on the continuation of antibiotics is shown on Figure 1.

Three days after the removal of glucocorticoids, the patient developed painful erythematous plaques on her trunk and also induration of the right forearm concerning for necrotizing fasciitis [Figure 2]. Surgical exploration of the arm was negative for signs of infection, but over the course of the next 12 hours the site of surgical trauma developed a sheet of coalescent pustules consistent with a pathergy response [Figure 3]. Punch biopsy of a trunk lesion showed a superficial, thin band of neutrophils with sparing of the epidermis and intact endothelium of the blood vessels consistent with neutrophilic dermatitis. Sweet's syndrome was diagnosed based on the constellation of biopsy findings, pathergy phenomenon and negative pathology and microbiology in a patient with underlying MDS. Prednisone $60 \mathrm{mg} / \mathrm{d}$ was started and within several days her fever and cutaneous lesions resolved, and she experienced dramatic global improvement. While the specific cause of the patient's initial upper airway obstruction is unknown, she attested to oral trauma from a food bolus the day her symptoms started. It is probable that this represented oral pathergy.

\section{Discussion}

\section{Diagnosis and Treatment}

This patient originally presented with upper airway obstruction, which was later found to be the initial symptom 
Table 2. Admission complete blood count, basic metabolic panel, and inflammatory markers. The thrombocytopenia was baseline for the patient due to her MDS.

\begin{tabular}{cc}
\hline Complete blood count & \\
\hline WBC & $4.1 \times 10^{3}$ \\
RBC & $3.5 \times 10^{3}$ \\
HGB & 9.8 \\
HCT & 28.9 \\
Platelets & $<10 \times 10^{3}$ \\
Segs (\%) & $53 \%$ \\
Blasts (\%) & $7 \%$ \\
\hline Basic metabolic panel & $178 \mathrm{mg} / \mathrm{dl}$ \\
\hline Glucose & $13 \mathrm{mg} / \mathrm{dl}$ \\
BUN & $0.3 \mathrm{mg} / \mathrm{dl}$ \\
Creatinine & $138 \mathrm{mEq} / \mathrm{L}$ \\
Sodium & $4.1 \mathrm{mEq} / \mathrm{L}$ \\
Potassium & $106 \mathrm{mEq} / \mathrm{L}$ \\
Chloride & $21 \mathrm{mEq} / \mathrm{L}$ \\
\hline CO & \\
\hline
\end{tabular}

Table 3. Inflammatory and autoimmune panels.

\begin{tabular}{cc}
\hline Sedimentation rate & $108 \mathrm{~mm} / \mathrm{hr}$ \\
C reactive protein & $26.8 \mathrm{mg} / \mathrm{dl}$ \\
ANA screen & Negative \\
DS DNA screen & Negative \\
ENA Smith & Negative \\
ENA RNP & Negative \\
Rheumatoid factor & Negative \\
SS A antibody & Negative \\
SS B antibody & Negative \\
C-ANCA & $<1: 20$ \\
P-ANCA & $<1: 20$ \\
C1 esterase inhibitor & 39 (within normal limits) \\
C3 complement & 103 (within normal limits) \\
C4 complement & 25 (within normal limits) \\
\hline
\end{tabular}




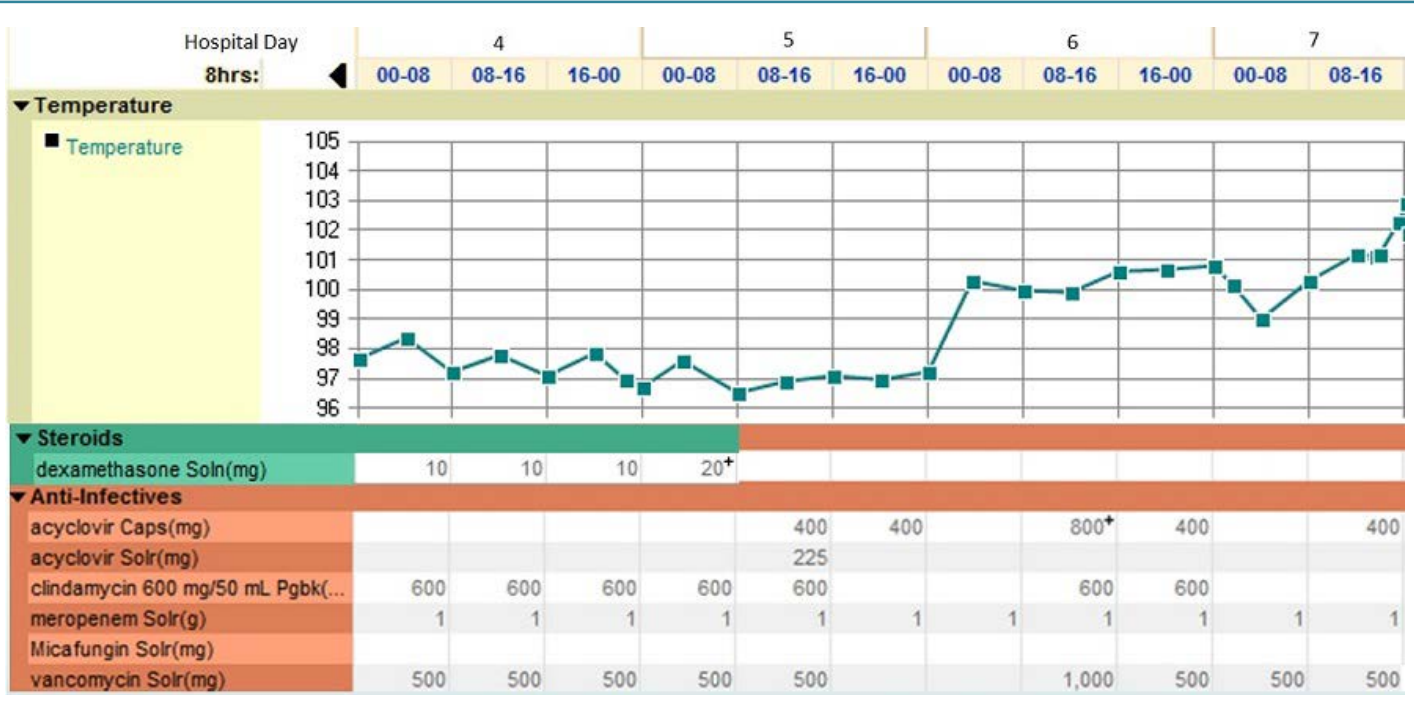

Figure 1. Return of previously treated fever with discontinuation of glucocorticoids.

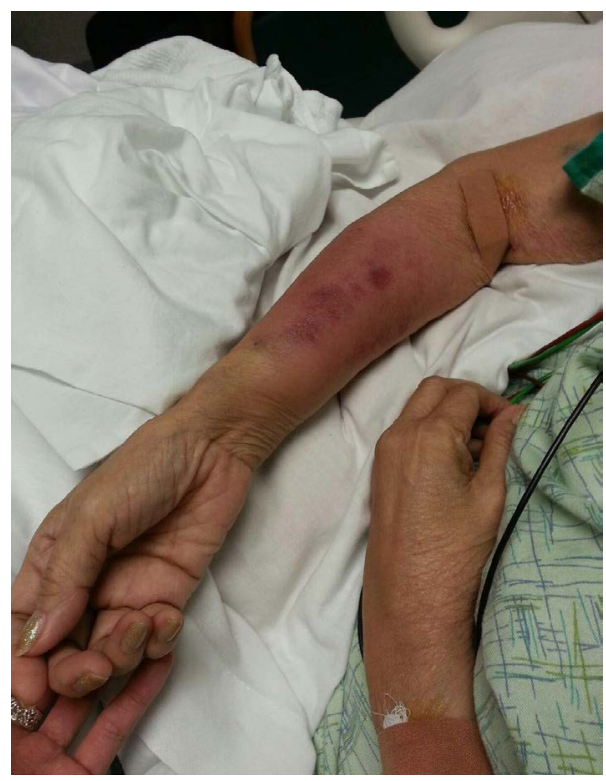

Figure 2. Patient's right arm, immediately preop.

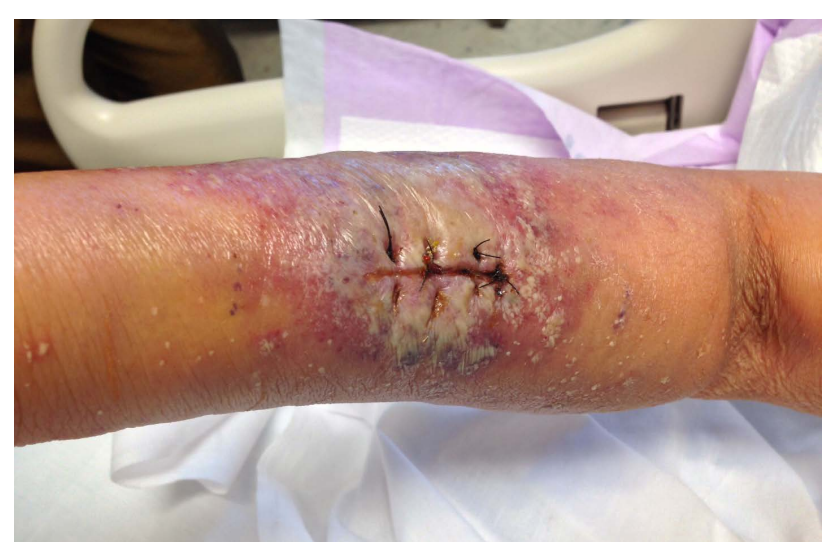

Figure 3. Patient's right arm, 12 hours postop. 
of Sweet's syndrome. At the time of presentation, there was no history of oral trauma, which made identifying the oral lesion a possible pathergy response diagnostically impossible. It was only after the patient developed pathergy to her surgical incision that the diagnosis became clear. With subsequent skin biopsy positive for neutrophilic infiltrate without leukocytoclastic vasculitis, characteristic truncal rash, hematologic malignancy, fever, and resolution of symptoms with glucocorticoids, she fit both major and three out of four minor criteria for Sweet's.

Sweet's syndrome can present with has a variety of clinical phenomena that are not a part of its diagnostic criteria of inflammation and a papular/nodular rash. Solid organ involvement has been seen including encephalitis [3] myocarditis, hepatitis, glomerulonephritis [4], and sterile osteomyelitis. Bullous Sweet's [5], subcutaneous Sweet's, and neutrophilic dermatosis of the dorsal hands [6] are common variants that present with atypical rash patterns. Nonspecific symptoms related to systemic inflammation such as malaise and diffuse pain are also commonly reported. Pathergy is uncommonly documented in Sweet's, but has a known association.

The pathergy response can be seen in a number of disorders, most notably Behcet's syndrome, where an inflammatory response to pinprick injury is part of the diagnostic criteria [7]. The response can be exuberant, and is often stimulated in response to minor or unnoticed trauma. While pathergy is not needed to diagnose Sweet's, it can offer a crucial clue as it did in this case. A detailed literature review found one other documented case of airway obstruction caused by pathergy in Sweet's syndrome, though in that case the inciting trauma was from a biopsy needle [8].

We chose to treat the patient with prednisone $60 \mathrm{mg} / \mathrm{d}$. Prednisone 0.5 - $1 \mathrm{mg} / \mathrm{kg} / \mathrm{d}$ is the standard dosing protocol for Sweet's syndrome to be continued for at least 4 - 6 weeks [9]. While colchicine and dapsone have also been shown anecdotally to be effective therapies, systemic glucocorticoids remain the gold standard. Success is assessed by rapid (<48 hours) resolution of fever and painful rash [10].

\section{Conclusion}

Making an initial diagnosis of Sweet's syndrome can be difficult due to its somewhat ambiguous presentation of systemic inflammation and rash, as well as the need for a skin biopsy. Without a very high index of suspicion, diagnosis can often be delayed resulting in patient morbidity. It is important for clinicians to recognize pathergy as a sign of Sweet's syndrome, and pursue a skin biopsy if new pathergy is observed in a patient with unexplained systemic inflammation. This is the first documented case of Sweet's syndrome presenting with oral pathergy.

\section{Acknowledgements}

Special thanks to Dr. John Snellings.

\section{References}

[1] Cohen, P.R. (2007) Sweet's Syndrome-A Comprehensive Review of an Acute Febrile Neutrophilic Dermatosis. Orphanet Journal of Rare Diseases, 2, 34. http://dx.doi.org/10.1186/1750-1172-2-34

[2] Raza, S., Kirkland, R., Patel, A., Shortridge, J. and Freter, C. (2013) Insight into Sweet’s Syndrome and AssociatedMalignancy: A Review of the Current Literature. International Journal of Oncology, 42, 1516-1522. http://dx.doi.org/10.3892/ijo.2013.1874

[3] Noda, K., Okuma, Y., Fukae, J., Fujishima, K., Goto, K., Sadamasa, H., Yoshiike, T. and Mizuno, Y. (2001) Sweet’s Syndrome Associated with Encephalitis. Journal of the Neurological Sciences, 188, 95-97. http://dx.doi.org/10.1016/S0022-510X(01)00541-X

[4] Lund, J.J., Stratman, E.J., Jose, D., Xia, L., Wilson, D. and Moizuddin, M. (2010) Drug-Induced Bullous Sweet Syndrome with Multiple Autoimmune Features. Autoimmune Diseases, 2010, Article ID: 176749. http://dx.doi.org/10.4061/2010/176749

[5] Voelter-Mahlknecht, S., Bauer, J., Metzler, G., Fierlbeck, G. and Rassner, G. (2005) Bullous Variant of Sweet’s Syndrome. International Journal of Dermatology, 44, 946-947. http://dx.doi.org/10.1111/j.1365-4632.2004.02287.x

[6] Walling, H.W., Snipes, C.J., Gerami, P. and Piette, W.W. (2006) The Relationship between Neutrophilic Dermatosis of the Dorsal Hands and Sweet Syndrome: Report of 9 Cases and Comparison to Atypical Pyoderma Gangrenosum. Archives of Dermatology, 142, 57-63. http://dx.doi.org/10.1001/archderm.142.1.57

[7] Levy, R., Junkins, J., Turchi, J. and James, W. (2002) Sweet Syndrome as the Presenting Symptom of Relapsed Hairy 
Cell Leukemia. Archives of Dermatology, 138, 1551. http://dx.doi.org/10.1001/archderm.138.12.1551

[8] Jo, T., Horio, K. and Migita, K. (2015) Sweet’s Syndrome in Patients with MDS and MEFV Mutations. New England Journal of Medicine, 372, 686-688. http://dx.doi.org/10.1056/NEJMc1412998

[9] Sakane, T., Takeno, M., Suzuki, N. and Inaba, G. (1999) Behçet’s Disease. New England Journal of Medicine, 341, 1284-1291. http://dx.doi.org/10.1056/NEJM199910213411707

[10] Bouw, J., Kater, A., Tongeren, J. and Schultz, M. (2007) Upper-Airway Obstruction Instigated by Sweet’s Syndrome. Medical Science Monitor: International Medical Journal of Experimental and Clinical Research, 13, CS53-CS55. 